\title{
LINE BROADENING IN AN AM MODE-LOCKED TEA-CO, LASER
}

\author{
R.J.M. BONNIE and F.A. VAN GOOR \\ Department of Applied Physics, Twente University of Technologv, Enschede, The Netherlands
}

Received 17 October 1985

By means of injection mode-locking, we were able to measure the bandwidth limited pulse duration of an AM mode-locked IEA-CO laser for different laser-gas mixtures. From these measurements we confirm the theoretical relation between bandwidth and pulse duration. The bandwidth under laser discharge conditions is similar to that of the neutral gas.

\section{Introduction}

Understanding and predicting the behaviour of a $\mathrm{CO}_{2}$ laser system requires among others an accurate knowledge of the bandwidth of the active medium. The most reliable and accurate expressions for the bandwidth of a $\mathrm{CO}_{2}$-laser gas mixture are obtained from absorption measurements under neutral conditions. They show that the bandwidth decreases with increasing helium percentage [1]. From this, it is clear that in the case of an AM modelocked TEA- $\mathrm{CO}_{2}$ laser, the duration of bandwidth limited pulses should increase with increasing helium percentage.

However, Witteman and Olbertz [2] reported a decreasing pulsewidth with increasing helium percentage. They concluded that under laser discharge conditions, the bandwidth may increase with increasing helium percentage. This intriguing observation is the reason for the present investigation. We studied the width of $\mathrm{CO}_{2}$ laser lines under discharge conditions by measuring the pulse width from an AM modelocked TEA-CO $\mathrm{CO}_{2}$ laser.

\section{Description of the experiment}

The technique we used for our investigation is in principle injection mode-locking [3]. The setup is shown in fig. 1.

The radiation from an $\mathrm{AM}$ mode-locked TEA- $\mathrm{CO}_{2}$ laser (master oscillator) is injected in a regenerative high pressure $\mathrm{TE}-\mathrm{CO}_{2}$ amplifier (slave). The master is formed by a single-discharge TEA-CO ${ }_{2}$ laser [4] with an active volume of $20 \times 1 \times 1 \mathrm{~cm}^{3}$ and an intra-cavity acousto-optic loss modulator. The slave oscillator is a UV preionized TE- $\mathrm{CO}_{2}$ laser [5] with an active volume of $30 \times 0.8 \times 0.8 \mathrm{~cm}^{3}$, operating at a pressure of 5 atmospheres. Due to pressure broadening the bandwidth of this amplifying medium is about $25 \mathrm{GHz}$, sufficiently large to avoid additional broadening or distortion of the injected pulses [6].

The delay between both discharges can be varied electronically. This delay could be measured very precisely by observing the light-signals coming from the last spark-gaps of the Marx generators of the lasers. The output from the master and slave oscillator is monitored via a photon-drag detector on a Tektronix $7912 \mathrm{AD}$ transient digitizer.

By varying the delay we were able to follow the pulse forming process in the master oscillator [7]. Making this delay sufficiently large, we would observe with this technique the width of the pulses in the stationary state of the AM mode-locking process. Keeping the ratio $\mathrm{CO}_{2}$ and $\mathrm{N}_{2}$ one to one, we measured in this way as a function of the helium percentage the duration of the bandwidth limited pulses. Because of a small staggering in the results, we averaged over a complete data set.

The final results are shown in fig. 2 . From this figure it is seen that increasing the fraction of helium in a $\mathrm{CO}_{2}$-laser leads to an enlarged steady-state pulse-width. 


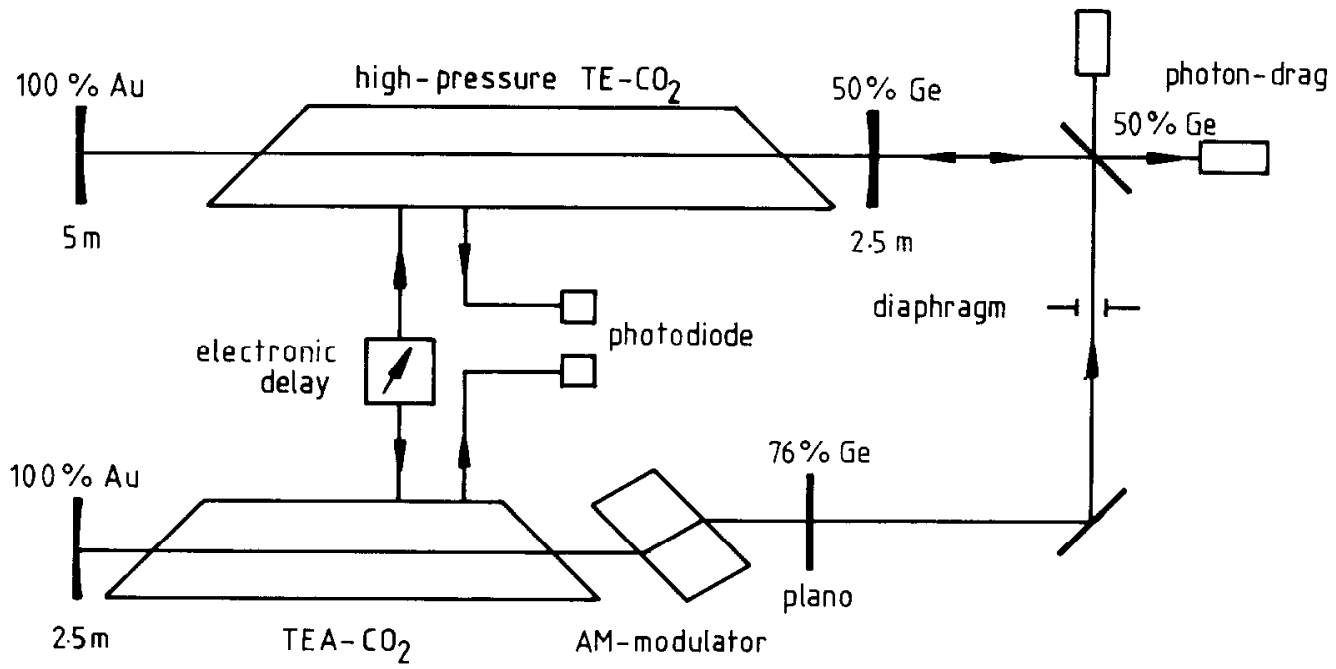

Fig. 1. Experimental setup.

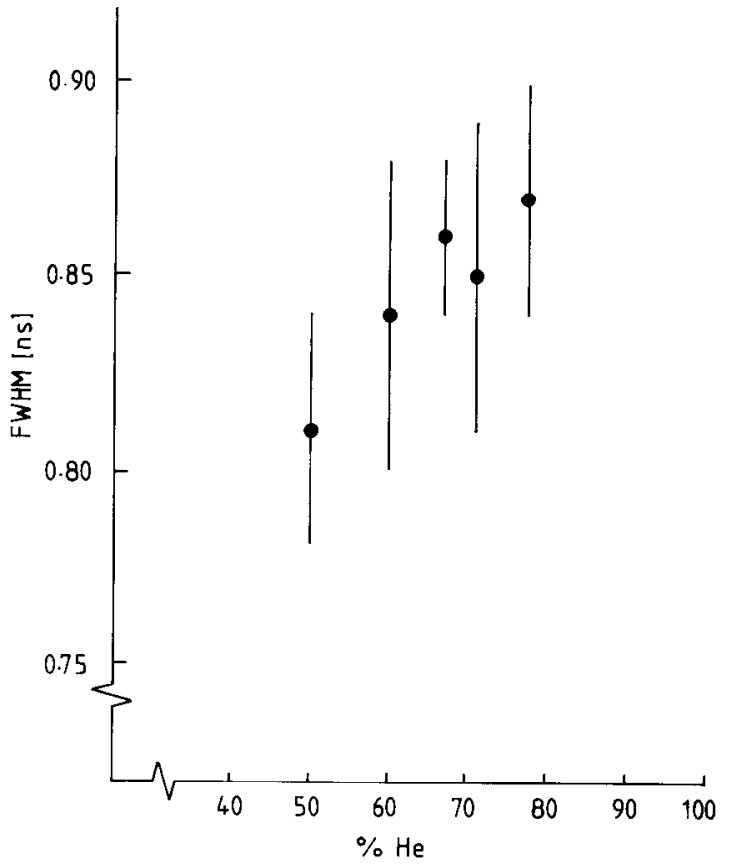

Fig. 2. Bandwidth limited pulse-width versus helium concentration in a mixture of $\mathrm{CO}_{2}: \mathrm{N}_{2}: \mathrm{He}=1: 1: n$.

\section{Determination of the bandwidth}

Applying the steady-state theory of Siegman and Kuizenga [8], the following expression for the bandwidth can be derived:

$\Delta \nu=\frac{\ln 2}{\pi^{2}}\left(\frac{-\ln R}{\delta_{\mathrm{m}}}\right)^{1 / 2} \frac{1}{f_{\mathrm{m}}} \frac{1}{\tau_{\mathrm{s}}^{2}}$,

where $R$ is the total cavity reflectivity, including other linear losses, $\delta_{\mathrm{m}}$ is the modulation depth of the AMmodulator, $f_{\mathrm{m}}$ is the modulator drive-frequency, $\tau_{\mathrm{s}}$ is the pulse width (FWHM) in steady state.

The results of our measurements according to (1) are presented in fig. 3 . The error-bars represent the spreading in the steady-state pulse-width. The contribution to the error due to the uncertainty in $R$ and $\delta_{\mathrm{m}}$ is not taken into account. Because we performed all measurements under identical experimental conditions, this part of the uncertainty results only in a small shift of the complete data set, and does not influence the position of the data-points relative to each other. The solid line in fig. 3 shows the bandwidth according to $[1]$ :

$\Delta \nu=7.58\left(\Psi_{\mathrm{CO}_{2}}+0.73 \Psi_{\mathrm{N}_{2}}+0.64 \Psi_{\mathrm{He}}\right) P(300 / T)^{1 / 2}$ 


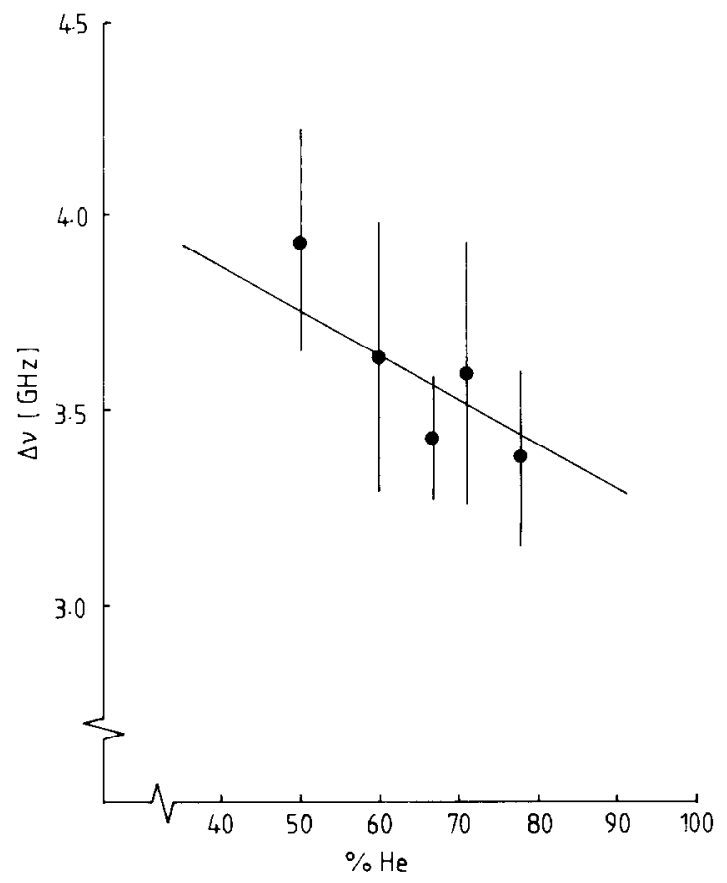

Fig. 3. Bandwidth according to eq. (1) for the data-set of fig. 2 . The solid line represents the bandwidth under neutral conditions, according to eq. (2).

where $[\Delta \nu]=\mathrm{MHz},[P]=$ Torr, $T$ is the (absolute) temperature, $\Psi_{i}$ is the fraction of component $i$ in the gas mixture.

\section{Discussion and conclusions}

Fig. 3 shows clearly that the relation between bandwidth and helium percentage under laser discharge conditions is very similar to the one under neutral conditions: bandwidth decreases with increasing helium percentage.

It will be clear from the above analysis that for ap- plying formula (1) it is very important that the pulse forming in the laser indeed reaches steady state. The results from ref. [2] can probably be understood from the fact that no steady state for the pulse forming was reached and so the measured pulse durations were not bandwidth limited.

Increasing the amount of helium results in a lower gain and so more roundtrips are needed before the gain switches. Therefore there is more time available for the pulse forming process to approach steady state. Hence shorter pulses occur in the gain switched peak by adding more helium. Summarizing we conclude that the bandwidth of a $\mathrm{CO}_{2}$ laser gas mixture under discharge conditions decreases with increasing heliumpercentage. The dependence of the bandwidth on this percentage under pulsed laser discharge conditions and under neutral conditions is very alike, in spite of the temperature rise during the discharge.

This investigation was made possible by the financial support of the Dutch "Stichting voor Fundamenteel Onderzoek der Materie".

\section{References}

[1] R.L. Abrams, Appl. Phys. Lett. 25 (1974) 609.

[2] W.J. Witteman and A.H.M. Olbertz, IEEE J. Quant. Electron. QE-13 (1977) 381.

[3] P.A. Bélanger and J. Boivin, Gigawatt peakpower pulse generation by injection of a single short pulse in a regenerative amplifier above threshold (RAAT).

[4] G.J. Ernst and A.G. Boer, Optics Comm. 34 (1980) 221.

[5] A.J. Alcock, K. Leopold and M.C. Richardson, Appl. Phys. Lett. 23 (1973) 562.

[6] F.A. van Goor, Optics Comm. 41 (1982) 205.

[7] F.A. van Goor, R.J.M. Bonnie and W.J. Witteman, IEEE J. Quant. Electon., to be published.

[8] J.J. Kuizenga and A.E. Siegman, IEEE J. Quant. Electron, QE-6 (1970) 694. 